\title{
Especies promisorias para el ecoturismo en "Campo Aventura Roca Madre", Toluviejo-Sucre, Colombia
}

\section{Promising species for the development of ecotourism in "Campo Aventura Roca Madre", Toluviejo-Sucre, Colombia}

\author{
Alcides Sampedro-M, ${ }^{1 *}$ Ph.D, Angel Álvarez-P, ${ }^{1}$ Biól, Luz M. Domínguez, ${ }^{1}$ Biól, \\ Ivonne Herrera-M, ${ }^{1}$ Biól.
}

\begin{abstract}
${ }^{1}$ Grupo de Biodiversidad Tropical, Universidad de Sucre, Sincelejo, Sucre, Colombia. *Correspondencia: asampedro2002@yahoo.es
\end{abstract}

Recibido: Febrero de 2011; Aceptado: Diciembre de 2011.

\section{RESUMEN}

Objetivo. Determinar las especies de flora y fauna más importantes para el desarrollo del ecoturismo, en una localidad del departamento de Sucre, Colombia. Materiales y métodos. Se hizo un listado de las especies de flora y fauna observadas en el sendero ecológico de un centro de ecoturismo en Toluviejo, Sucre y se calculó su abundancia, importancia económica, estado de conservación y adaptaciones, que fueron evaluadas mediante un índice de importancia potencial para el ecoturismo, que suma los valores que se asignan a las variables consideradas de interés. Resultados. Se detectaron 18 especies promisorias de fauna, de 91 especies observadas y 16 especies vegetales de 53 observadas. Los animales de mayor potencial ecoturístico resultantes, fueron: Dendrobates truncatus y Saguinus oedipus, especies endémicas de Colombia, seguidas por Basiliscus basiliscus, Atta sp., Morpho peleides, Lycorea halia y Ara ararauna; todas estas con una alta abundancia y fácil detección. Dentro de las plantas, Anacardium excelsum obtuvo el mayor índice de importancia, como resultado de su endemismo y sus adaptaciones. Las especies Piper auritum, Matayba escrobiculata, Tabernaemontana cymosa y la comúnmente conocida como jazmín de monte, fueron fácilmente detectables y abundantes, además Brownea ariza y Tabebuia rosea resultaron las más representativas por la belleza de sus flores. Conclusiones. Se determinaron las especies que pueden ser de interés para los ecoturistas y esto hace posible mejorar el producto que se oferta a partir de profundizar en su conocimiento.

Palabras clave: Ecoturismo sostenible, especies promisorias, fauna silvestre, flora (Fuente: Tesauro ambiental para Colombia). 


\begin{abstract}
Objectives. To determine the species of flora and fauna relevant for development of ecotourism in a location of Sucre, Colombia. Materials and methods. A list of various species at the ecotourism center in the municipality of Toluviejo, Sucre, was studied which included abundance, economic and conservation relevance and adaptation data. The census was estimated and evaluated according to our index of potential importance for ecotourism, by adding the values assigned to each of the variables of interest. Results. 18 promising species of fauna and 16 promising species of plants were found. Animals with higher ecotourism potential were Dendrobates truncatus and Saguinus oedipus, endemic to Colombia, followed by Basiliscus basiliscus, Atta sp. Morpho peleides, Lycorea halia, Ara ararauna, all these of high abundance and easy detection. Regarding plants, Anacardium excelsum obtained the highest value as a result of endemism and their adaptations. Piper auritum, Matayba escrobiculata, Tabernaemontana cymosa and the specie commonly known as "jazmín de monte", were easily detectable and abundant. Brownea ariza and Tabebuia rosea were the best candidates because of the beauty of their flowers. Conclusions. The species that may be of interest to eco-tourists were determined and this makes it possible to improve the product offering from deepening their knowledge.
\end{abstract}

Key words: Flora, promising species, sustainable ecotourism, wildlife (Source: Tesauro ambiental para Colombia).

\section{INTRODUCCION}

En Colombia se ha prestado atención oficial al ecoturismo a través de un documento conjunto entre los Ministerios de Comercio, Industria y Turismo y el Ministerio de Vivienda y Desarrollo Territorial (1) en el que se traza la Política Para el Desarrollo del Ecoturismo. En este documento se enfoca la necesidad de capacitar a las personas, profesionales o no, acerca del conocimiento ambiental, como única forma de lograr una conservación efectiva. Berovides (2) y Rodríguez y Berovides (3) propusieron una metodología, que valora la fauna promisoria para el ecoturismo, lo que permite brindar un producto de calidad al ecoturista y compatibilizar el uso de la fauna con su conservación. Los autores en mención se basaron en que los productos que brinda el ecoturismo se relacionan con la belleza que se observa en la naturaleza y las características ecológicas de los organismos que allí habitan.

El objetivo del presente trabajo fue hacer un inventario de las especies principales de flora y fauna en el área que ocupa "Campo Aventura Roca Madre" en el departamento de Sucre, Colombia. Además, se determinó cuáles de ellas tienen mayor importancia para la actividad ecoturística, dándole valor a la importancia de las diferentes especies, teniendo en cuenta la abundancia, lo endémico de la especie, la belleza y las posibilidades de ser observadas, entre otros elementos (2).

\section{MATERIALES Y MÉTODOS}

Área de estudio. La serranía de Coraza y Montes de María, bajo la jurisdicción de CARSUCRE (4), forma parte de la serranía de San Jacinto. Situada al norte del departamento de Sucre, en áreas compartidas por los municipios de Colosó, Chalán y Toluviejo, y abarca una extensión de 6.653 ha. Allí se encuentra "Campo Aventura Roca Madre", localizado en el corregimiento de La Piche $\left(09^{\circ} 30^{\prime} 45,73^{\prime \prime} \mathrm{N}\right.$ y $075^{\circ} 24^{\prime} 37,12^{\prime \prime}$ W), municipio de Toluviejo, con una extensión de 127 ha. Este sitio se caracteriza por poseer una cobertura de bosque seco tropical, formadas por superficies montañosas de elevación mediana, con alturas que van desde los 200 a los 560 msnm, temperatura promedio de $27^{\circ} \mathrm{C}$, y con precipitación promedio anual de $1500 \mathrm{~mm}$.

Procedimiento para la determinación de especies promisorias. La metodología (3) contempla diez elementos a evaluar que son: 1) accesibilidad al hábitat de la especie, 2) si es o no endémica, 3 ) su detectabilidad (si es posible observarla con facilidad o si es difícil por su coloración, porque se oculta, o por alguna otra razón), 4) la abundancia, 5) actividad diaria, 6) actividad estacional, 7) adaptaciones, 8) utilización por el hombre, 9) situación conservacionista y 10) valor sistemático. Este último, en el presente trabajo se modificó por "aspecto" de la especie, debido a se consideró el interés que expresan los ecoturistas en relación a si un animal es grande o pequeño, si presenta un color llamativo, o se camufla muy bien entre la vegetación por su coloración críptica, si tiene un dimorfismo sexual marcado. En el caso de las 
plantas, se tuvo la misma consideración, ya que estas pueden llamar la atención por su altura, grosor, cobertura, presencia de espinas, hojas grandes, coloración, entre otras características. En el caso de la flora se sustituyó "actividad estacional" por "caducidad".

A cada parámetro se le asignó valores entre uno y diez, en función de su mayor o menor importancia respecto a los otros. Al valor así obtenido se le denomina Índice de Potencialidad Ecoturística Ponderado ( $\operatorname{IPE}_{w}$ ) (2). Para ello, se acordó un criterio único sobre el orden de importancia de los elementos considerados; este acuerdo parte del principio de que siempre el valor de los elementos es subjetivo y variable en relación a la la localidad y la especie a evaluar, pero se puede obtener consenso de cuáles son aquellos que motivan a los ecoturistas y establecer un orden de prioridad que permita cuantificar las preferencias.

En el caso de la fauna los valores asignados fueron:
1) Si la especie es endémica 10
2) Abundancia 9
3) Detectabilidad 8
4) Accesibilidad al hábitat 7
5) Adaptaciones 6
6) Aspecto 5
7) Actividad diaria 4
8) Actividad estacional 3
9) Peligro de extinción 2
10) Valor etnozoológico 1

Para la fauna, la suma de los valores de cada elemento es 55 como valor máximo y el valor promedio más uno, es decir $27.5+1$, es el límite de selección de una especie considerada promisoria.

En el caso de la flora los valores asignados fueron:
1) Si la especie es endémica 9
2) Abundancia 8
3) Detectabilidad 7
4) Accesibilidad al hábitat 6
5) Adaptaciones 5
6) Aspecto 4
7) Caducidad 3
8) Peligro de extinción 2
9) Valor etnobotánico 1

Para la flora se procedió de la misma manera, pero en este caso la suma total es 45 y el límite de selección de una especie considerada promisoria es $22.5+1$.
Trabajo de campo. Este trabajo se realizó entre los meses de febrero y julio del 2010, finalizando el período seco y comenzando el período de Iluvias y durante este tiempo se desarrollaron 54 salidas de campo de 8 horas cada una. Para observar la fauna se utilizaron binoculares, telescopio Tele Star y cámara fotográfica Cannon Rebel XS con Zoom acoplado. En el caso de la flora, las observaciones se hicieron mediante inspección ocular.

Las visitas se realizaron en la mañana y en la tarde al sendero ecológico, que tenía una extensión de $2069 \mathrm{~m}$ y el ancho que se tuvo en cuenta a ambos lados, para las observaciones, fue de $20 \mathrm{~m}$. La posición geográfica al inicio del sendero es $9^{\circ} 30^{\prime} 41.2^{\prime \prime} \mathrm{N}$ y $75^{\circ} 24^{\prime} 37.8^{\prime \prime} \mathrm{O}$, con una altura de $91 \mathrm{~m}$, y al final $9^{\circ} 30^{\prime} 47.0^{\prime \prime}$ $\mathrm{N}$ y $75^{\circ} 23^{\prime} 54.6^{\prime \prime} \mathrm{O}$, con una altura de $288 \mathrm{~m}$, aunque la altura mayor en este sendero fue de $321 \mathrm{~m}$. Se trata de un sector donde abundan las pequeñas y medianas elevaciones y arroyos y se observa cierto grado de antropización, sobre todo al inicio y al final del trayecto, debido a la presencia de animales domésticos en las cercanías y a la influencia de una cortadora de mármol, también cercana; sin embargo, la vegetación y la fauna características del lugar fueron predominantes siempre.

Se anotaban las especies de flora y fauna observadas y la hora de observación. Las especies vegetales se identificaron directamente en el lugar y las que no era posible identificar, se les tomó muestras de hojas y frutos para su posterior análisis y consulta con especialistas y la bibliografía (5). El mismo procedimiento se utilizó con la fauna observada y en este caso la bibliografía utilizada fue fundamentalmente para aves (6).

A partir de las observaciones directas y del análisis bibliográfico acerca de las especies observadas, se obtuvieron las principales adaptaciones, morfológicas, fisiológicas y conductuales, así como el establecimiento de la actividad diaria y estacional de cada especie, determinando la partición del recurso tiempo, en base a las horas de avistamiento durante todo el trabajo de campo y a lo hallado en la literatura. Los datos acerca de la utilización de la flora y la fauna se obtuvieron a partir de la bibliografía especializada y a través de entrevistas aleatorias con este fin, a 65 personas adultas (más de 25 años), de ambos sexos (38 hombres y 27 mujeres).

La abundancia relativa de las principales especies de fauna fue medida como la frecuencia de avistamiento: 
$\mathrm{F}=\mathrm{N}^{\circ}$ de avistamiento de cada especie / $\mathrm{N}^{\circ}$ de muestreos por 100

Se determinó la abundancia absoluta de las principales especies de plantas en el sendero ecológico, como:

$$
A=N^{\circ} \text { de individuos /área total }
$$

El área del sendero ecológico es de 8.3 ha. Los resultados se interpretaron teniendo en cuenta el valor mínimo y máximo de la abundancia, de este modo se tomaron rangos como sigue: 1-20 poco observada (PO); 21-40 moderadamente común (Mc); 41-70 común (C); 71-100 abundante (A).

\section{RESULTADOS}

En la tabla 1 aparece el listado de las plantas observadas en el parque durante el período de trabajo. Se trata de 29 familias y 53 especies.

En la tabla 2 se registran las especies de fauna observadas en el área de estudio. Aparecen 49 familias y 91 especies, dentro de las que, las aves son las mejor representadas con 46 especies, repartidas en 23 familias. Las familias que mostraron mayor riqueza de especies fueron Psittacidae y Accipitridae, con 8 y 4, respectivamente.

Tabla 1. Especies de flora observadas en el sendero ecológico de Aventura Roca Madre.

\begin{tabular}{|c|c|c|}
\hline FAMILIA & ESPECIE & NOMBRE COMÚN \\
\hline Acanthaceae & Tichanthera sp & palo de agua \\
\hline \multirow[t]{3}{*}{ Anacardiaceae } & Anacardium excelsum & caracolí \\
\hline & Mangifera indica & mango \\
\hline & Spondias mombin & jobo \\
\hline \multirow[t]{4}{*}{ Apocynaceae } & Aspidosperma polyneuron & carreto \\
\hline & Tabernaemontana cymosa & bola de barraco \\
\hline & Tabernaemontana sp & tomatón \\
\hline & Plumeria alba & azuceno \\
\hline Aquifoliaceae & Ilex sp & barbasco de monte \\
\hline \multirow[t]{3}{*}{ Arecaceae } & Desmoncus orthacanthos & matamba \\
\hline & Sabal mauritiiformis & palma amarga \\
\hline & Attalea butyracea & palma de vino \\
\hline \multirow[t]{2}{*}{ Araceae } & Alocasia macrorrhiza & malanga \\
\hline & Dieffenbachia sp & matapuerco \\
\hline \multirow[t]{4}{*}{ Bignoniaceae } & Crescentia cujete & totumo \\
\hline & Macfadyena uncata & uña de gato \\
\hline & Tabebuia rosea & roble \\
\hline & Tabebuia chrysea & polvillo \\
\hline \multirow[t]{3}{*}{ Bombacaceae } & Ceiba pentandra & ceiba de agua \\
\hline & Bombacopsis quinata & ceiba tolúa \\
\hline & Pseudobombax septenatum & majagua \\
\hline Burseraceae & Bursera simarouba & indio en cuero o almácigo \\
\hline Cecropiaceae & Cecropia obtusifolia & guarumo \\
\hline Combretaceae & Sp indeterminada & varaleòn \\
\hline Elaeocarpaceae & Muntingia calabura & periquito \\
\hline Eritroxilaceae & Erythoxylon sp & coca de mico \\
\hline Euphorbiaceae & Hura crepitans & ceiba de leche \\
\hline Flacourtiaceae & Casearia corymbosa & vara de humo \\
\hline Heliconiaceae & Heliconia sp & lengüeta \\
\hline Lauraceae & Persea americana & aguacate \\
\hline Lecythidaceae & Gustavia superba & membrillo \\
\hline \multirow[t]{3}{*}{ Leguminosa/ Caesalpinaceae } & Brownea ariza & arizal \\
\hline & Caesalpinia coriaria & dividivi \\
\hline & Haematoxylum brasiletto & brasil \\
\hline \multirow[t]{4}{*}{ Leguminosa/ Fabaceae } & Albizia saman & campano \\
\hline & Albizia niopoides & guacamayo \\
\hline & Calliandra magdalenae & carbonero \\
\hline & Zygia inaequalis & guamo macho \\
\hline
\end{tabular}




\begin{tabular}{lll}
\hline Leguminosa/ papilionoideae & Platymiscium pinnatum & trébol \\
Meliaceae & Cedrela odorata & cedro \\
& Brosimum alicastrum & guáimaro \\
& Ficus sp & caucho \\
& Ficus dendroica & suán \\
Piperaceae & Piper auritum maría \\
Rubiaceae & Sp indeterminada & jazmín de monte \\
Sapindaceae & Simira cordifolia & pijiño \\
& Talisia olivaeformis & mamón de mico \\
Sapotaceae & Matayba escrobiculata & guacharaco \\
Sterculiaceae & Manilkara zapota & níspero \\
& Guazuma ulmifolia & guácimo \\
Urticaceae & Sterculia apétala & camajón \\
Verbenaceae & Urera baccifera & pringamoza \\
\hline
\end{tabular}

Tabla 2. Especies de fauna observadas en el sendero ecológico de Campo Aventura Roca Madre.

\begin{tabular}{|c|c|c|}
\hline FAMILIA & ESPECIE & NOMBRE COMÚN \\
\hline \multicolumn{3}{|c|}{ INVERTEBRADOS } \\
\hline Formicidae & Atta sp. & hormiga arriera \\
\hline Morphidae & Morpho peleides & mariposa azul \\
\hline Nymphalidae & Lycorea halia & mariposa \\
\hline \multicolumn{3}{|c|}{ VERTEBRADOS } \\
\hline \multicolumn{3}{|c|}{ ANFIBIOS } \\
\hline Bufonidae & Bufo marinus & sapo común \\
\hline Dendrobatidae & Dendrobates truncatus & rana venenosa \\
\hline Hylidae & Hyla crepitans & rana platanera \\
\hline \multicolumn{3}{|c|}{ REPTILES } \\
\hline \multirow[t]{3}{*}{ Anomalepididae } & Liotyphlops albirostris & ciega \\
\hline & Leptodeira septentrionalis & mapaná \\
\hline & Liophis lineatus & guardacamino \\
\hline \multirow[t]{5}{*}{ Colubridae } & Oxybelis aeneus & bejuquillo \\
\hline & Mastigodryas boddaerti & cazadora \\
\hline & Pseudoboa neuwiedii & candelilla roja \\
\hline & Urotheca euryzonus & coral falsa \\
\hline & Helicops danieli & mapaná de agua \\
\hline Corytophanidae & Basiliscus Basiliscus & pasarroyo \\
\hline \multirow{2}{*}{ Gekkonidae } & Hemidactylus brooki & lagartija \\
\hline & Gonatodes albogularis & salamanqueja \\
\hline Gymnophthalmidae & Tretioscincus bifasciatus & lagartija cola azul \\
\hline Iguanidae & Iguana iguana & iguana \\
\hline Polychrotidae & Anolis vittigerus & lagartija \\
\hline \multirow[t]{2}{*}{ Scincidae } & Mabuya mabouya & lisa \\
\hline & Tupinambis teguixin & lobo pollero \\
\hline \multirow[t]{2}{*}{ Teiidae } & Ameiva festiva & lobito \\
\hline & Ameiva Ameiva & lobito común \\
\hline \multirow[t]{2}{*}{ Viperidae } & Porthidium nasutum & patoco \\
\hline & Bothrops asper & mapaná negra \\
\hline & AVES & \\
\hline \multirow[t]{4}{*}{ Accipitridae } & Buteogallus meridionalis & bebehumo \\
\hline & Buteogallus urubutinga & águila negra \\
\hline & Buteo magnirostris & gavilán jabao \\
\hline & Leucopternis albicollis & águila blanca \\
\hline Aramidae & Aramus guarauna & carrao \\
\hline Caprimulgidae & Nyctidromus albicollis & bujío \\
\hline \multirow[t]{3}{*}{ Cathartidae } & Sarcoramphus papa & rey golero \\
\hline & Cathartes aura & aura \\
\hline & Coragyps atratus & golero \\
\hline \multirow[t]{3}{*}{ Columbidae } & Columba fasciata & paloma \\
\hline & Zenaida auriculata & paloma torcaza \\
\hline & Columbina talpacoti & tortolita tierrera \\
\hline \multirow[t]{2}{*}{ Cracidae } & Penelope purpurascens & pava congona \\
\hline & Ortalis garrula & guacharaca \\
\hline Curvidae & Cyanacorax affinis & charán o chau chau \\
\hline \multirow[t]{2}{*}{ Cuculidae } & Crotophaga ani & chingola \\
\hline & Crotophaga mayor & cocinera \\
\hline \multirow[t]{3}{*}{ Falconidae } & Falco peregrines & halcón \\
\hline & Falco sparverius & halcón o cernícalo \\
\hline & Milvago chimachima & pígua \\
\hline
\end{tabular}




\begin{tabular}{|c|c|c|}
\hline \multirow[t]{2}{*}{ Fringillidae } & Oryzoborus crassirostris & yolofo \\
\hline & Sicalis flaveola & canario \\
\hline Galbulidae & Galbula ruficauda & jacamar \\
\hline Hirumdidae & Progne chalybea & golondrina \\
\hline \multirow[t]{2}{*}{ Icteridae } & Psarocolius decumanus & oropendola \\
\hline & Agelaius icterocephalus & toche \\
\hline Mimidae & Mimus gilvus & sinsonte \\
\hline Parulidae & Troglodytes aedo & cucarachero común \\
\hline Picidae & Campephilus melanoleucos & carpintero moña roja \\
\hline \multirow[t]{8}{*}{ Psittacidae } & Aratinga pertinax & cotorra cara sucia \\
\hline & Ara ararauna & guacamaya azul \\
\hline & Ara macao & guacamaya bandera \\
\hline & Ara severa & guacamaya verde \\
\hline & Amazona ochrocephala & Ioro copete amarillo \\
\hline & Amazona amazónica & Ioro basto \\
\hline & Brotogeris ugularis & perico \\
\hline & Pionus menstruus & periquito cabeciazul \\
\hline Ramphastidae & Ramphastus sulfuratus & guasalé \\
\hline Strigidae & Bubo virginianus & búho real \\
\hline \multirow[t]{3}{*}{ Thraupidae } & Thraupis palmarum & azulejo palmero \\
\hline & Thraupis episcopus & azulejo común \\
\hline & Ramphocelus diminiatus & sangre toro \\
\hline Trochiladae & Anthracothorax nigricolis & chupaflor \\
\hline Troglodytidae & Campylorhynchus griseus & chupa huevo \\
\hline \multirow[t]{2}{*}{ Tyrannidae } & Elaenia flavogaster & copetón \\
\hline & Megarhynchus pitangua & chuchafria \\
\hline \multicolumn{3}{|c|}{ MAMIFEROS } \\
\hline Agoutidae & Agouti paca & guartinaja \\
\hline \multirow[t]{2}{*}{ Atelidae } & Alouatta seniculus & mono aullador colorado \\
\hline & Ateles fusciceps & marimonda \\
\hline \multirow[t]{2}{*}{ Cebidae } & Saguinus oedipus & tití cabeza blanca \\
\hline & Cebus capucinus & mono capuchino \\
\hline Dasypodidae & Dasypus novemcinctus & armadillo \\
\hline Dasyproctidae & Dasyprocta punctata & ñeque \\
\hline Didelphidae & Didelphis marsupialis & zorra chucha \\
\hline Felidae & Leopardus pardales & tigrillo \\
\hline Sciuridae & Sciurus granatensis & ardilla roja \\
\hline
\end{tabular}

Tabla 3. Valor de uso y conservacionista de la fauna en Campo Aventura Roca Madre.

\begin{tabular}{|c|c|c|c|c|c|c|c|}
\hline Especie & C & UIA & M & $\mathbf{P}$ & $\mathbf{E}$ & $\mathbf{C A}(7,8)$ & CITES \\
\hline Morpho peleides & & $X(9)$ & & & & & \\
\hline Dendrobates truncates $(10)$ & & & & $x$ & $x$ & LR:Ca & AP II \\
\hline Bufo marinus(11) & & $\mathrm{x}$ & & & & & \\
\hline Iguana iguana(12) & $x$ & $x$ & $\mathrm{x}$ & & & & AP II \\
\hline Tupinambis teguixin(13) & $x$ & $x$ & & & & & AP II \\
\hline Astigodryas boddaerti & & & & $x$ & & & \\
\hline Leptodeira septentrionalis & & & & $x$ & & & \\
\hline Liophis lineatus & & & & $x$ & & & \\
\hline Ramphocelus dimidiatus & & & $X(14)$ & & & & \\
\hline Columba fasciata(15) & $x$ & & $x$ & & & & \\
\hline Penelope purpurascens(16) & $x$ & & & & & & AP III \\
\hline Ortalis garrula(16) & $x$ & & $\mathrm{x}$ & & $x$ & & \\
\hline Ramphastus sulfuratus & & & $x$ & & & & AP II \\
\hline Ara ararauna(17) & & $x$ & $x$ & & & & AP II \\
\hline Ara severa & & $X(17)$ & $X(18)$ & & & & AP II \\
\hline Amazona farinosa & & $x$ & $x$ & & & & AP II \\
\hline Amazona ochrocephala & & $X(17)$ & $X(18)$ & & & & AP II \\
\hline Brotogeris jugularis & & & $X(18)$ & & & & AP II \\
\hline Buteogallus meridionalis & & & & & & & AP II \\
\hline Buteo magnirostris & & & & & & & AP II \\
\hline Milvago chimachima & & & & & & & AP II \\
\hline Alouatta seniculus & $X(18)$ & & $x$ & & & LR/VU & AP II \\
\hline Saguinus oedipus & $X(18)$ & & & & $x$ & & AP I \\
\hline Cebus capucinus & & & $\mathrm{x}$ & & & LR:Ca & AP II \\
\hline Sciurus granatensis & $X(18)$ & & $x$ & & & & \\
\hline
\end{tabular}

C: comestible; UIA: uso industrial y artesanal; M: uso como mascota; P: peligrosa; E: endémica; CA: categoría de amenaza; VU: Vulnerable; LR: Bajo riesgo, Ca: Casi amenazado. 
Tabla 4. Valor de uso y conservacionista de la flora en Campo Aventura Roca Madre

\begin{tabular}{|c|c|c|c|c|c|c|c|c|}
\hline Especie & $\begin{array}{l}\text { Prop. } \\
\text { curativas }\end{array}$ & c & $\mathbf{T}$ & M & E & $\begin{array}{l}\text { Uso } \\
\text { industrial } \mathrm{y} / \mathrm{o} \text { artesanal }\end{array}$ & $\begin{array}{l}\text { Uso } \\
\text { ornamental }\end{array}$ & $\mathbf{C A}(19)$ \\
\hline Hura crepitan(20) & $\mathrm{x}$ & & $\mathrm{x}$ & $\mathrm{x}$ & & $\mathrm{x}$ & $\mathrm{x}$ & \\
\hline Ceiba pentandra(20) & $\mathrm{x}$ & $\mathrm{x}$ & & $\mathrm{x}$ & & $\mathrm{x}$ & $\mathrm{x}$ & LC \\
\hline Anacardium excelsum(20) & & & & $\mathrm{x}$ & $\mathrm{x}$ & $\mathrm{x}$ & & LC/NT \\
\hline Brownea ariza(21) & $\mathrm{x}$ & & & $\mathrm{x}$ & & $\mathrm{x}$ & & \\
\hline Tabernaemontana cymosa & & & & $\mathrm{x}$ & & & $\mathrm{x}$ & \\
\hline Guazuma ulmifolia(20) & $\mathrm{x}$ & & & $\mathrm{x}$ & & & & \\
\hline Tabebuia rosea(20) & $\mathrm{x}$ & & & $\mathrm{x}$ & & & $\mathrm{x}$ & \\
\hline Sterculia apétala(20) & $\mathrm{x}$ & $\mathrm{x}$ & & $\mathrm{x}$ & & $\mathrm{x}$ & $\mathrm{x}$ & \\
\hline Sabal mauritiiformis(20) & & $\mathrm{x}$ & & & & $\mathrm{x}$ & $\mathrm{x}$ & NT \\
\hline Platymiscium pinnatum(20) & $\mathrm{x}$ & & & $\mathrm{x}$ & & & $\mathrm{x}$ & \\
\hline Pseudobombax septenatum(20) & & & & $\mathrm{x}$ & & $\mathrm{x}$ & $\mathrm{x}$ & \\
\hline Alocasia macrorrhiza(22) & & $\mathrm{x}$ & $\mathrm{x}$ & & & $\mathrm{x}$ & $\mathrm{x}$ & \\
\hline Spondias mombin(20) & $\mathrm{x}$ & $\mathrm{x}$ & & & & & & \\
\hline Bombacopsis quinata(23) & & & & $\mathrm{x}$ & & $\mathrm{x}$ & & \\
\hline Bursera simarouba(20) & $\mathrm{x}$ & & & & & $\mathrm{x}$ & & \\
\hline Piper auritum(24) & $\mathrm{x}$ & & & & & & & \\
\hline Sp. Indeterminada1 (Jazmin de monte) & & & $\mathrm{x}$ & & & & $\mathrm{x}$ & \\
\hline Dieffenbachia sp.(25) & & & $\mathrm{x}$ & & & & $\mathrm{x}$ & \\
\hline Brosimum alicastrum(20) & $\mathrm{x}$ & $\mathrm{x}$ & & $\mathrm{x}$ & & $\mathrm{x}$ & $\mathrm{x}$ & \\
\hline Tabebuia chrysea(20) & & & & $\mathrm{x}$ & & $\mathrm{x}$ & & NT \\
\hline Cecropia obtusifolia(26) & $\mathrm{x}$ & & & $\mathrm{x}$ & & & & \\
\hline Sp. Indeterminada 2 (Varaleon) & & & & & & & & \\
\hline
\end{tabular}

C: Comestible, T: Tóxica, M: Maderable, E: Endémica. Casi amenazado (NT), Preocupación menor (LC), En peligro (EN).

El valor de uso y conservacionista para las especies que presentaban alguno de ellos, aparece en las tablas 3 y 4 , para fauna y flora respectivamente. La mayor parte de las especies de fauna presentan algún valor de uso, sobre todo como mascota y algunas comestibles. Solo tres de ellas son endémicas y la mayor parte aparecen en el apéndice II de CITES. La flora por su parte, presenta un gran valor de uso de todo tipo, hay pocas especies endémicas y solo cuatro con algún grado de amenaza.

Sólo siete especies resultaron abundantes, dentro de las cuales el mono aullador, las hormigas arrieras y la mariposa azul, fueron avistadas en todos los muestreos (Tabla 5). El lobito común, una mariposa, el pasarroyo y el chau chau, fueron las otras especies abundantes. En cuanto a la flora, tres especies aparecen con mayor abundancia absoluta, el jazmín de monte, la santa maría y el caracolí (Tabla 6).

La gran mayoría de las especies que fueron observadas en Campo aventura Roca Madre son activas durante las horas del día (Tabla 7) y solo tres son migratorias.

Las especies de fauna que por el valor que obtuvieron del índice de potencialidad ecosturística ponderado (IPEW) se pueden considerar promisoras, aparecen en la Tabla 8.

De las especies evaluadas, 18 presentaron IPE superior a 28.5, por lo que se seleccionan como
Tabla 5. Abundancia relativa de la fauna en Campo Aventura Roca Madre.

\begin{tabular}{|c|c|c|}
\hline ESPECIE & AR & $\mathbf{I}$ \\
\hline Atta sp. & 100 & A \\
\hline Morpho peleides & 100 & A \\
\hline Alouatta seniculus & 100 & A \\
\hline Ameiva ameiva & 92.5 & A \\
\hline Lycorea halia & 81.5 & A \\
\hline Basiliscus basiliscus & 77.8 & A \\
\hline Cyanocorax affinis & 77.8 & A \\
\hline Dendrobates truncatus & 66.7 & C \\
\hline Ramphastus sulfuratus & 63.0 & C \\
\hline Ara ararauna & 51.9 & C \\
\hline Sciurus granatensis & 51.9 & C \\
\hline Anolis vittigerus & 44.4 & C \\
\hline Amazona farinosa & 44.4 & C \\
\hline Amazona ochrocephala & 44.4 & C \\
\hline Ara severa & 33.3 & Mc \\
\hline Iguana iguana & 22.2 & Mc \\
\hline Leptodeira meridionalis & 22.2 & Mc \\
\hline Penelope purpurascens & 22.2 & Mc \\
\hline Ortalis garrula & 22.2 & Mc \\
\hline Sarcoramphus papa & 22.2 & Mc \\
\hline Buteo magnirostris & 22.2 & Mc \\
\hline Saguinus oedipus & 22.2 & Mc \\
\hline Campephilus melanoleucos & 18.5 & $\mathrm{PO}$ \\
\hline Tupinambis teguixin & 14.8 & $\mathrm{PO}$ \\
\hline Cebus capucinus & 14.8 & $\mathrm{PO}$ \\
\hline Bufo marinus & 11.1 & $\mathrm{PO}$ \\
\hline Mastigodryas boddaerti & 11.1 & $\mathrm{PO}$ \\
\hline Liophis lineatus & 11.1 & $\mathrm{PO}$ \\
\hline Columba fasciata & 11.1 & $\mathrm{PO}$ \\
\hline Brotogeris jugularis & 11.1 & $\mathrm{PO}$ \\
\hline Cathartes aura & 11.1 & $\mathrm{PO}$ \\
\hline Milvago chimachima & 11.1 & $\mathrm{PO}$ \\
\hline Ramphocelus dimidiatus & 7.4 & $\mathrm{PO}$ \\
\hline Elaenia flavogaster & 7.4 & $\mathrm{PO}$ \\
\hline Psarocolius decumanus & 7.4 & $\mathrm{PO}$ \\
\hline Crotophaga ani & 7.4 & $\mathrm{PO}$ \\
\hline Buteogallus meridionalis & 7.4 & $\mathrm{PO}$ \\
\hline
\end{tabular}

AR: abundancia relativa; I: interpretación; A: abundante; C: común; MC: Moderadamente común; PO: poco observada 
Tabla 6. Abundancia absoluta de la flora detectada en el sendero ecológico de Campo Aventura Roca Madre.

\begin{tabular}{|c|c|}
\hline ESPECIE & $\begin{array}{c}\text { ABUNDANCIA } \\
\text { ABSOLUTA } \mathrm{ni} / \mathrm{ha}\end{array}$ \\
\hline sp.indeterminada 1 (jazmín de monte) & 58.1 \\
\hline Piper auritum & 23.6 \\
\hline Anacardium excelsum & 21.3 \\
\hline Talisia olivaeformis & 8.7 \\
\hline Matayba escrobiculata & 8.2 \\
\hline Tabernaemontana cymosa & 7.6 \\
\hline Dieffenbachia sp & 7.6 \\
\hline Brownea ariza & 5.2 \\
\hline Hura crepitans & 4.5 \\
\hline Alocasia macrorrhiza & 3.3 \\
\hline Sp. Indeterminada 2 (varaleón) & 3.1 \\
\hline Tabebuia rosea & 2.8 \\
\hline Ficus sp & 2.7 \\
\hline Sabal mauritiiformis & 2.7 \\
\hline Tabernamontana sp & 2.7 \\
\hline Spondias mombin & 2.0 \\
\hline Guazuma ulmifolia & 1.7 \\
\hline Sterculia apetala & 1.6 \\
\hline Simira cordifolia & 1.4 \\
\hline Pseudobombax septenatum & 1.3 \\
\hline Ilex sp & 1.0 \\
\hline Zygia inaequalis & 0.7 \\
\hline Brosimum alicastrum & 0.7 \\
\hline Ceiba pentandra & 0.4 \\
\hline Tabebuia chrysea & 0.2 \\
\hline Platymiscium pinnatum & 0.2 \\
\hline Cecropia obtusifolia & 0.1 \\
\hline
\end{tabular}

promisorias para el ecoturismo en la localidad de estudio. Los mayores valores los posee Dendrobates truncatus y Saguinus oedipus, especies endémicas de Colombia, seguidas por Basiliscus basiliscus, Atta sp., Morpho peleides, Lycorea halia, Ara ararauna, todas con alta abundancia y detectabilidad. Las mariposas y la guacamaya azul, presentaron además un gran atractivo por su belleza. Por otra parte, 19 especies resultaron con valores por debajo de 28.5, por lo que no se consideran apropiadas para el ecoturismo.

De las 27 especies vegetales evaluadas (Tabla 9), 16 presentaron un IPEw superior a 25.5, Anacardium excelsum obtuvo el valor más alto como resultado de su endemismo y sus adaptaciones. Las especies Piper auritum, Matayba escrobiculata, Tabernaemontana cymosa y la comúnmente conocida como jazmín de monte, fueron fácilmente detectables y abundantes, además Brownea ariza
Tabla 7. Partición del recurso tiempo (diario y estacional) para la fauna de Campo Aventura Roca Madre.

\begin{tabular}{|c|c|c|c|c|c|}
\hline \multirow[t]{2}{*}{ ESPECIE } & \multicolumn{3}{|c|}{ Actividad diaria } & \multicolumn{2}{|c|}{$\begin{array}{l}\text { Actividad } \\
\text { estacional }\end{array}$} \\
\hline & D & c & $\mathbf{N}$ & $\mathbf{R}$ & $\mathbf{M}$ \\
\hline Atta sp.(27) & $\mathrm{x}$ & & & $\mathrm{x}$ & \\
\hline Morpho peleides(5) & $\mathrm{x}$ & & & $\mathrm{x}$ & \\
\hline Lycorea halia(5) & $\mathrm{x}$ & & & $\mathrm{x}$ & \\
\hline Dendrobates truncates(6) & $x$ & & & $x$ & \\
\hline Bufo marinus(8) & & $x$ & $\mathrm{x}$ & $\mathrm{x}$ & \\
\hline Basiliscus basiliscus(28) & $\mathrm{x}$ & & & $x$ & \\
\hline Anolis vittigerus(29) & $\mathrm{x}$ & & & $\mathrm{x}$ & \\
\hline Iguana iguana(10) & $\mathrm{x}$ & & & $\mathrm{x}$ & \\
\hline Ameiva ameiva & $\mathrm{x}$ & & & $\mathrm{x}$ & \\
\hline Tupinambis teguixin(11) & $\mathrm{x}$ & & & $\mathrm{x}$ & \\
\hline Mastigodryas boddaerti(11) & & & $\mathrm{x}$ & $\mathrm{x}$ & \\
\hline Leptodeira septentrionalis & & $\mathrm{x}$ & $x$ & $\mathrm{x}$ & \\
\hline Liophis lineatus(11) & $\mathrm{x}$ & & & $\mathrm{x}$ & \\
\hline Ramphastus sulfuratus & $\mathrm{x}$ & & & $\mathrm{x}$ & \\
\hline Amazona ochrocephala(15) & $x$ & & & $x$ & \\
\hline Amazona farinosa & $x$ & & & $x$ & \\
\hline Cyanocorax affinis & $\mathrm{x}$ & & & $\mathrm{x}$ & \\
\hline Ara ararauna(15) & $\mathrm{x}$ & & & $\mathrm{x}$ & \\
\hline Ara severa(15) & $\mathrm{x}$ & & & $\mathrm{x}$ & \\
\hline Brotogeris jugularis(15) & $x$ & & & $x$ & \\
\hline Ramphocelus dimidiatus(12) & $x$ & & & $\mathrm{x}$ & \\
\hline Elaenia flavogaster(12) & $\mathrm{x}$ & & & $\mathrm{x}$ & \\
\hline Psarocolius decumanus(12) & $\mathrm{x}$ & & & $\mathrm{x}$ & \\
\hline Campephilus melanoleucos(12) & $\mathrm{x}$ & & & $\mathrm{x}$ & \\
\hline Columba fasciata(13) & $x$ & & & $x$ & \\
\hline Crotophaga ani & & & & $x$ & \\
\hline Penelope purpurascens(14) & $\mathrm{x}$ & & & $\mathrm{x}$ & \\
\hline Ortalis garrula(14) & $x$ & & & $x$ & \\
\hline Sarcoramphus papa(30) & $\mathrm{x}$ & & & & $\mathrm{x}$ \\
\hline Cathartes aura(30) & $x$ & & & & $x$ \\
\hline Buteogallus meridionalis(30) & $x$ & & & & $x$ \\
\hline Buteo magnirostris(29) & $\mathrm{x}$ & & & $x$ & \\
\hline Milvago chimachima(30) & $\mathrm{x}$ & & & $\mathrm{x}$ & \\
\hline Cebus capucinus & $\mathrm{x}$ & & & $\mathrm{x}$ & \\
\hline Saguinus oedipus & $\mathrm{x}$ & & & $\mathrm{x}$ & \\
\hline Alouatta seniculus(5) & $x$ & & & $\mathrm{x}$ & \\
\hline Sciurus granatensis(31) & $\mathrm{x}$ & & & $\mathrm{x}$ & \\
\hline
\end{tabular}

$\mathrm{D}$ : diurno; C: crepuscular; $\mathrm{N}$ : nocturno; R: residente; $\mathrm{M}$ : migratorio

y Tabebuia rosea fueron las más representativas por la belleza de sus flores, y alrededor del $40 \%$ son caducifolias o semicaducifolias, que las hacen características de este tipo de bosque. También se puede notar en esta tabla que tres de las especies promisorias para el ecoturismo, entre ellas, Sabal mauritiformis y Bombacopsis quinata, presentaron problemas de conservación, lo que demuestra que su uso en el ecoturismo debe ser sustentado con un plan de manejo bien estructurado. 
Tabla 8. Índice de potencialidad ecoturística ponderado (IPEw), de las especies de fauna consideradas promisorias en Campo Aventura Roca Madre. El número entre paréntesis se corresponde con el valor máximo que tiene ese elemento.

\begin{tabular}{|c|c|c|c|c|c|c|c|c|c|c|c|}
\hline ELEMENTOS/ ESPECIES & $E(10)$ & $A B(9)$ & $\mathrm{D}(\mathbf{8})$ & $\mathrm{AH}(7)$ & $\mathrm{AD}(6)$ & $A(5)$ & ADI(4) & $\mathrm{AE}(3)$ & $\operatorname{sc}(2)$ & VE(1) & TOTAL \\
\hline Dendrobates truncatus & 10 & 9 & 8 & 7 & 6 & 5 & 4 & 3 & 0 & 0 & 52 \\
\hline Basiliscus basiliscus & 0 & 9 & 8 & 7 & 6 & 2 & 4 & 3 & 2 & 1 & 42 \\
\hline Anolis vittigerus & 0 & 5 & 4 & 6 & 5 & 3 & 4 & 3 & 2 & 0 & 32 \\
\hline Ameiva meiva & 0 & 8 & 7 & 6 & 3 & 2 & 4 & 3 & 2 & 0 & 35 \\
\hline Cyanocorax affinis & 0 & 8 & 8 & 2 & 4 & 2 & 4 & 3 & 2 & 0 & 33 \\
\hline Ara ararauna & 0 & 8 & 8 & 2 & 4 & 5 & 4 & 3 & 2 & 1 & 37 \\
\hline Ara severa & 0 & 7 & 8 & 2 & 4 & 5 & 4 & 3 & 0 & 1 & 34 \\
\hline Ramphastus sulfuratus & 0 & 8 & 8 & 2 & 5 & 5 & 4 & 3 & 0 & 1 & 36 \\
\hline Amazona farinosa & 0 & 7 & 7 & 2 & 5 & 5 & 4 & 3 & 0 & 1 & 34 \\
\hline Amazona ochrocephala & 0 & 7 & 7 & 2 & 5 & 5 & 4 & 3 & 0 & 1 & 34 \\
\hline Ortalis arrula & 10 & 3 & 1 & 2 & 4 & 2 & 3 & 3 & 0 & 1 & 29 \\
\hline Sciurus granatensis & 0 & 7 & 7 & 2 & 6 & 5 & 4 & 3 & 0 & 1 & 35 \\
\hline Saguinus oedipus & 10 & 6 & 7 & 2 & 6 & 5 & 4 & 3 & 0 & 1 & 44 \\
\hline Alouatta seniculus & 0 & 7 & 8 & 2 & 6 & 4 & 4 & 3 & 0 & 1 & 35 \\
\hline Cebus capucinus & 0 & 6 & 7 & 2 & 6 & 5 & 4 & 3 & 0 & 1 & 34 \\
\hline Atta ephalotes & 0 & 9 & 8 & 7 & 6 & 2 & 4 & 3 & 2 & 0 & 41 \\
\hline Lycorea halia & 0 & 7 & 8 & 6 & 4 & 5 & 4 & 3 & 2 & 1 & 40 \\
\hline Morpho peleides & 0 & 7 & 8 & 6 & 4 & 5 & 4 & 3 & 2 & 1 & 40 \\
\hline
\end{tabular}

E: endemismo; AB: abundancia; D: detectabilidad; AH: accesibilidad al hábitat; AD: adaptaciones; A: aspecto; ADI: actividad diaria; AE: actividad estacional; SC: situación conservacionista; VE: valor etnozoológico.

Tabla 9. Índice de potencialidad ecoturística ponderado (IPEW), de las especies de flora consideradas promisorias en Campo Aventura Roca Madre. El número entre paréntesis se corresponde con el valor máximo que tiene ese elemento.

\begin{tabular}{|c|c|c|c|c|c|c|c|c|c|c|}
\hline ELEMENTOS/ ESPECIES & $E(9)$ & $A B(8)$ & $\mathrm{D}(7)$ & $A H(6)$ & $A D(5)$ & $\mathrm{B}(4)$ & DE(3) & $\operatorname{SC}(2)$ & VE(1) & TOTAL \\
\hline Sp. Indeterminada (jazmín de monte) & 0 & 8 & 7 & 6 & 3 & 3 & 0 & 2 & 1 & 30 \\
\hline Piper auritum & 0 & 8 & 7 & 6 & 3 & 2 & 0 & 2 & 1 & 29 \\
\hline Anacardium excelsum & 9 & 8 & 7 & 6 & 5 & 4 & 0 & 0 & 1 & 40 \\
\hline Tabernaemontana cymosa & 0 & 6 & 6 & 6 & 3 & 2 & 0 & 2 & 1 & 26 \\
\hline Dieffenbachia sp & 0 & 6 & 6 & 6 & 5 & 4 & 0 & 2 & 1 & 30 \\
\hline Brownea ariza & 0 & 4 & 7 & 6 & 4 & 4 & 0 & 2 & 1 & 28 \\
\hline Hura crepitans & 0 & 4 & 7 & 6 & 4 & 4 & 3 & 2 & 1 & 31 \\
\hline Alocasia macrorrhiza & 0 & 4 & 6 & 6 & 4 & 4 & 0 & 2 & 1 & 27 \\
\hline Tabebuia rosea & 0 & 4 & 7 & 6 & 5 & 4 & 3 & 0 & 1 & 30 \\
\hline Ficus sp & 0 & 4 & 7 & 6 & 4 & 3 & 0 & 2 & 1 & 27 \\
\hline Spondias mombin & 0 & 3 & 6 & 6 & 3 & 3 & 3 & 2 & 1 & 27 \\
\hline Guazuma Imifolia & 0 & 2 & 7 & 6 & 4 & 3 & 2 & 2 & 1 & 27 \\
\hline Sterculia apétala & 0 & 2 & 7 & 6 & 5 & 4 & 3 & 2 & 1 & 30 \\
\hline Pseudobombax septenatum & 0 & 2 & 7 & 6 & 4 & 4 & 3 & 2 & 1 & 29 \\
\hline Ceiba pentandra & 0 & 1 & 7 & 6 & 5 & 4 & 2 & 0 & 1 & 26 \\
\hline Bombacopsis quinata & 0 & 1 & 7 & 6 & 5 & 4 & 3 & 0 & 1 & 27 \\
\hline
\end{tabular}

Endemismo; AB: abundancia; D: detectabilidad; AH: accesibilidad al hábitat; AD: adaptaciones; B: belleza; DE: deciduasd diaria; SC: situación conservacionista; VE: valor etnobtánico.

\section{DISCUSIÓN}

La flora y fauna reportadas en la zona de estudio resultan coincidentes con los reportes hechos en los últimos años por investigadores de esta región en localidades relativamente cercanas, como Colosó (32-34), lo que pudiera significar que también en esa y otras localidades cercanas la fauna promisoria para el ecoturismo coincidiría con la que se reporta en este trabajo. No obstante, sería necesario realizar estudios similares en las diferentes localidades, porque pueden existir diferencias notables entre las condiciones ambientales de cada sitio, así como diferencias en las formas de uso del suelo por el hombre y esto pudiera influir en los elementos que se evalúan y dar como resultado otras especies promisorias.
Las especies aquí avaladas como de mayor importancia para el ecoturismo, pueden ser tratadas como tal, utilizando los resultados para promocionar los centros de ecoturismo y las especies seleccionadas que servirían para atraer mayor público y realizar una labor de educación ambiental más efectiva, con información detallada e interesante de aquellas especies que observarán. Otro aspecto a considerar es, que las especies endémicas y las que presentan algún grado de amenaza, deben ser consideradas cuidadosamente en los planes de ecoturismo, de manera que su supervivencia sea asegurada (2).

Para algunas especies que no obtuvieron el puntaje necesario para quedar dentro de las promisorias, pero que son de interés por su gran abundancia, características adaptativas o 
belleza, deberá hacerse un análisis más detallado y particular (2). Estas especies podrían ser de interés para muchas personas. Tal es el caso de Milvago chimachima, un gavilán que se observa con mucha frecuencia y se ve claramente durante su vuelo de búsqueda de presas emitiendo silbidos de alta intensidad (35); lo mismo sucede con Campephilus melanoleucos, pájaro carpintero, de gran tamaño, de color negro con líneas blancas y copete rojo que llama mucho la atención por su canto característico y su forma peculiar de buscar alimento taladrando con su fuerte pico en el tronco de los árboles (6); por último está el caso de Bufo marinus, un sapo de gran tamaño, que se infla como una pelota cuando es atacado por sus enemigos y que puede aparecer en pleno día, cercano a cuerpos de agua. Además, su cuerpo está cubierto de glándulas venenosas, de las cuales se extraen sustancias que se utilizan en farmacología, como la bufotoxina (36). Esto evidencia que la metodología descrita y utilizada puede ser subjetiva, son los mismos investigadores los que determinan cuáles son los aspectos de mayor o menor valor, no obstante, un análisis detallado de las características de cada localidad donde se aplique, permitiría determinar las especies más importantes en esa localidad específica (3).
También debe considerarse el hecho de que pudieran concurrir visitantes que tienen mayor interes por una especie en específico; tal es el caso de los turistas científicos, que son mayormente profesores universitarios, científicos, naturalistas, que viajan a determinados sitios en el mundo para corroborar información científica, desarrollar proyectos o con otros intereses. En estos casos, las especies promisorias pueden no ser exactamente el objetivo que persiguen y el nivel de información que los mismos tienen es diferente al de la población común, por tanto deberá pensarse en una atención especializada donde intervengan profesionales de la temática de interés en cada caso.

Esta metodología puede ser utilizada, con algunas modificaciones, para determinar "sitios" adecuados para el ecoturismo, lo que ha sido demostrado para arrecifes coralinos (3) y esto puede ser de importancia para la planificación científica de los lugares de mayor interés para el ecoturismo, tanto a nivel departamental, como nacional.

\section{REFERENCIAS}

1. Ministerio de Comercio, Industria y Turismo, Ministerio de Vivienda y Desarrollo Territorial. Política para el desarrollo del ecoturismo. Bogotá, República de Colombia 2003.

2. Berovides V. Método de valoración de la fauna para el ecoturismo. Universidad de La Habana, Rev Biol 2002; 14(2):108- 113.

3. Rodríguez R, Berovides V. Evaluación de taxocenosis animales para el uso en el ecoturismo. Universidad de La Habana, Rev Biol 2003; 17(1):42-46.

4. Vásquez VH. Reservas Forestales Protectoras Nacionales de Colombia, Atlas Básico. Conservación Internacional Colombia; Ministerio de Ambiente, Vivienda y Desarrollo Territorial; Embajada Real de los Países Bajos. Bogotá, Colombia 2005.

5. Gentry AH. Diversity and floristic composition of neotropical dry forest. En Tropical Deciduous Forest Ecosystem. Bullock S. Medina E. Mooney HA. (Eds). Cambridge Univ: Press, Cambridge; 1995.
6. Steven H, Brow W. Guía de las Aves de Colombia. Bogotá: Asociación Colombiana de Ornitología; Cargraphics S. A., 2009.

7. Rueda-Almonacid JV, Lynch JD, Amézquita A. (Eds). Libro rojo de anfibios de Colombia. Serie Libros rojos de Especies Amenazadas de Colombia. Bogotá, Colombia: Conservación Internacional Colombia, Instituto de Ciencias Naturales, Universidad Nacional de Colombia, Ministerio del Medio Ambiente; 2004.

8. Rodríguez M, Alberico JVM., Trujillo F, Jorgenson J. (Eds.). Libro Rojo de los Mamíferos de Colombia. Serie Libros Rojos de Especies Amenazadas de Colombia. Bogotá, Colombia: Conservación Internacional Colombia y Ministerio de Ambiente, Vivienda y Desarrollo Territorial; 2006.

9. López-Sorto RE. Estado actual del conocimiento de las mariposas de El Salvador. Costa Rica: Instituto Nacional de Biodiversidad de Costa Rica. Santo Domingo de Heredia; 2007. 
10. Barrero C. Evaluación del efecto del extracto de alcaloides de Dendrobates truncatus (Anura: Dendrobatidae) sobre unión neuromuscular de mamífero. Bogotá, Colombia: Pontificia Universidad Javeriana. Facultad de Ciencias; 2005.

11. De Urioste J, Bethencourt M. Rana toro y sapo marino: la amenaza que viene. Revista de Medio Ambiente. Fundación Neotrópico $2001 ; 20: 2$.

12. Sánchez $\mathrm{CH}$, Castaño MO, Cárdenas AG. Diversidad de reptiles en Colombia. Bogotá, Colombia: Universidad Nacional de Colombia, Instituto de Ciencias Naturales; 1995.

13. Rodríguez JV, Rueda JV, Gutiérrez TD. Guía ilustrada de la fauna del Santuario de Vida Silvestre Los Besotes, Valledupar, Cesar, Colombia. Serie de guías tropicales de campo No 7. Conservación Internacional. Bogotá, Colombia: Editorial Panamericana, Formas e Impresos; 2008.

14. Hilty S, Brown W. Guía de Aves de Colombia. New Jersey: Princeton University Press; 2001.

15. Villareal J. Alternativas para el manejo de la paloma collareja Columba fasciata en el Área de Conservación Amistad Pacífico, Costa Rica. Área de Conservación Amistad Pacifico (Ministerio de Ambiente y Energía). San José, Costa Rica: Asociación Costarricense de Pesca, Tiro y Caza, Instituto Nacional de Biodiversidad; 1999.

16. Rodríguez J, Hughes N, Nieto D, Franco A. Paujiles, Pavones, Pavas y Guacharacas neotropicales. Bogotá, Colombia: Conservación Internacional; 2005.

17. Rodríguez J, Rojas F, Arzuza D, Gonzales A. Loros, Pericos y Guacamayas Neotropicales. Bogotá, Colombia: Conservación Internacional; 2005.

18. Racero J, Vidal C, Ruiz O, Ballesteros J. Percepción y patrones de uso de la fauna silvestre por las comunidades indígenas Embera- Katíos en la cuenca del río San Jorge zona amortiguadora de PNN Paramillo. Rev Est Soc 2008; 3:118-131.
19. Cárdenas LD, Salinas NR. (Eds.). Libro Rojo de Plantas de Colombia. Volumen 4. Especies maderables amenazadas: Primera parte, Serie Libros rojos de especies amenazadas de Colombia, Bogotá. Instituto Amazónico de Investigaciones Científicas SINCHI- Ministerio de Ambiente, Vivienda y Desarrollo Territorial: 2007.

20. Barbosa C, Ruíz CA, García H, Gutiérrez TD. Guía ilustrada de plantas destacadas del Santuario de Vida Silvestre Los Besotes, Valledupar, Cesar, Colombia. Con descripciones y anotaciones sobre distribución, aspectos ecológicos y usos locales. Serie de guías tropicales de campo No 8. Conservación Internacional. Bogotá, Colombia: Editorial Panamericana, Formas e Impresos; 2008.

21. Cárdenas D, Ramírez J. Plantas útiles y su incorporación a los sistemas productivos del departamento del Guaviare (Amazonía colombiana). Caldasia 2004; 26(1):95-110.

22. Gómez M, Acero L. Guía para el cultivo y el aprovechamiento del Bore Alocasia macrorrhiza. Convenio Andrés Bello Ministerio de Educación y Cultura de España. Fundación Centro para la Investigación en Sistemas Sostenibles de Producción Agropecuaria (CIPAV) 2002.

23. Cordero J, Boshier D. (Eds). Árboles de Centroamérica: un manual para extensionistas. Costa Rica: Centro Agronómico Tropical de Investigación y enseñanza (CATIE); 2003.

24. Blanco N, Ramos A, Vizoso A. Evaluación tóxica y genotóxica del extracto fluido de Piper auritum H.B.K. Instituto Superior de Ciencias Médicas de La Habana Facultad de Medicina "Dr. Salvador Allende". Revista Cubana Plantas Medicinales 2006; 11:3-4.

25. Coté G. Diversity and distribution of idioblasts producing calcium oxalate crystals in Dieffenbachia seguine (Araceae). Am Jour Bot 2009; 1:10-17.

26. Carvajal S, González L. La familia Cecropiaceae en el estado de Jalisco. México: Universidad de Guadalajara; 2005.

27. Fernández F. Introducción a las hormigas de la región Neotropical. Bogotá, Colombia: Instituto de Investigación de Recursos Biológicos "Alexander Von Humboldt"; 2003. 
28. Rand A, Marx H. Running speed of the Lizard Basiliscus on water. Copeia 1967; 1:230-233.

29. Navarro P, Garrido O. Especie nueva de Anolis (Sauria: Lacertilia: Iguanidae) de la región Suroriental de Cuba: Solenodon 2004; 4:85-90.

30. Márquez C, Bechard M, Vanegas V, Gast F. Aves rapaces diurnas de Colombia: Instituto de Investigación de Recursos Biológicos "Alexander Von Humboldt" 2005.

31. Espinel M. (2007): Comportamiento de las ardillas Sciurus granatensis y Microsciurus pucheranii en cautiverio antes y después de la implementación de un plan de enriquecimiento ambiental. Bogotá, Colombia: Pontificia Universidad Javeriana; 2007.

32. Galván S, De la Ossa J. Herpetofauna registrada para el área de influencia de la Reserva Forestal Protectora Serranía de Coraza, Colosó, Sucre, Colombia. Rev Colombiana Cienc Anim 2009; 1(2): 50-58.
33. Galván S, Sierra MI, Gómez H, De La Ossa J, Fajardo A. Biodiversidad en el área de influencia de la Estación Primates de Colosó, Sucre, Colombia. Rev Colombiana Cien Anim 2009; 1(1): 98-121.

34. Galván S. Mamíferos y aves silvestres registradas en una zona de los Montes de María, Colosó, Sucre, Colombia. Rev Colombiana Cienc Anim 2010; 2(1): 45-57.

35. De La Ossa-V J, De la Ossa-L A. Aspectos de la densidad poblacional e historia natural de Milvago chimachima (AVES: Falconidae) en el área urbana de Sincelejo (Sucre, Colombia). Univ Sci 2011; 16(1): 63-69.

36. Hutchinson D. Savitzky A. Vasculature of the Parotoid Glands of Four Species of Toads (Bufonidae: Bufo). Virginia. J Morphol 2004; (260):247-254. 